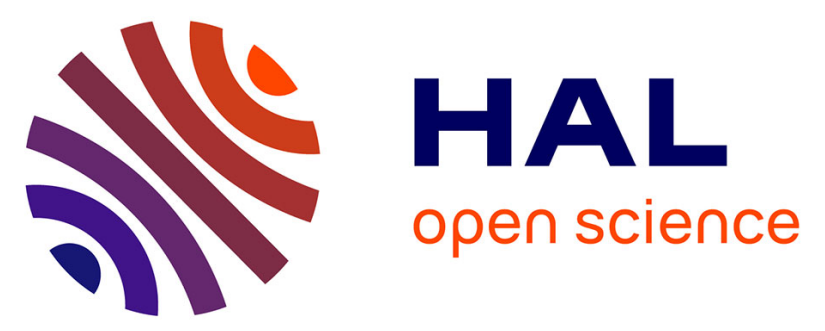

\title{
Enseigner en LVR une structure syntaxique en régression : quels enjeux, quelle méthode? Le cas de la réduplication en miroir en créole
}

Béatrice Jeannot-Fourcaud

\section{- To cite this version:}

Béatrice Jeannot-Fourcaud. Enseigner en LVR une structure syntaxique en régression: quels enjeux, quelle méthode? Le cas de la réduplication en miroir en créole. Contextes et Didactiques, 2014, Grammaires créoles, 4. hal-02050106

\section{HAL Id: hal-02050106 https://hal.univ-antilles.fr/hal-02050106}

Submitted on 26 Feb 2019

HAL is a multi-disciplinary open access archive for the deposit and dissemination of scientific research documents, whether they are published or not. The documents may come from teaching and research institutions in France or abroad, or from public or private research centers.
L'archive ouverte pluridisciplinaire HAL, est destinée au dépôt et à la diffusion de documents scientifiques de niveau recherche, publiés ou non, émanant des établissements d'enseignement et de recherche français ou étrangers, des laboratoires publics ou privés.

\section{다)(1) $\$$}

Distributed under a Creative Commons Attribution - NonCommerciall 4.0 International 


\title{
Enseigner en LVR une structure syntaxique en régression : Quels enjeux, quelle méthode? Le cas de la réduplication en miroir en créole
}

\author{
Béatrice JEANNOT-FOURCAUD \\ Université des Antilles et de la Guyane - CRREF, EA 4538
}

\section{Résumé}

Cet article est centré sur l'examen d'une structure de réduplication particulière, la réduplication en miroir, attestée en créole martiniquais et guadeloupéen notamment, mais qui apparaît désormais d'un usage limité. Cette structure se présente formellement comme la réduplication de l'unité verbale et la co-référence d'un pronom personnel. A partir d'une recherche menée auprès d'une population d'élèves guadeloupéens, nous aborderons cette structure selon trois points de vue : descriptif, sociolinguistique et didactique. Nous nous intéresserons particulièrement à la vitalité de cette structure au sein de la population à l'étude et envisagerons les pistes possibles pour son enseignement. Ce travail s'inscrit dans la problématique plus large de la place à accorder, dans le cadre de l'enseignement des LVR, à des structures syntaxiques en régression dans les usages.

\section{Mots-clés}

Enseignement des LVR, créole, syntaxe, réduplication, structure syntaxique en régression.

\begin{abstract}
This article deals with a Creole structure of reduplication, which is still used in Martinique and Guadeloupe but seems to be in decline now. This structure, I have named Réduplication en miroir, is characterized by the repetition of the verbal unit and the presence of a personal pronoun that comes after it. Based on a research within a population of pupils inhabitants of Guadeloupe, this article considers this structure through descriptive, sociolinguistic and didactic points of view. We are particularly interested in evaluating its vitality within the observed population and in making some proposals for its teaching. This work is part of a broader issue concerning the level of priority to give to declining syntactic structures within the framework of regional languages teaching.
\end{abstract}

\section{Keywords}

Teaching of regional languages, Creole, syntax, reduplication, declining syntactic structure. 


\section{Introduction}

Dans cet article, nous nous intéresserons à une structure de réduplication ${ }^{1}$ particulière, attestée en créoles martiniquais et guadeloupéen notamment, et impliquant non seulement la réduplication de l'unité verbale mais également la co-référence* du pronom personnel, comme dans les exemples qui suivent ${ }^{2}$ :

(1)Lésé'y alé alé'a-y.

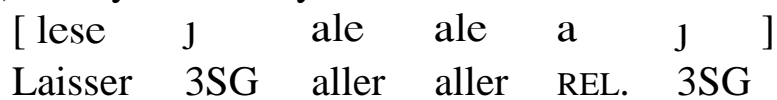

«Laisse-le aller à sa guise. »

(2)Nou té ka domi domi an-nou lè yo soné.

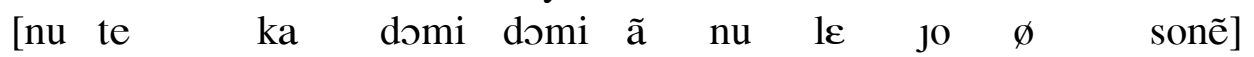

1PL PASSE INAC dormir dormir REL. 1PL quand 3SG ACC téléphoner

« Nous dormions tout notre saoul lorsqu'ils ont téléphoné. »

Cette structure qui apparaît à l'heure actuelle d'un usage limité, sera abordée de fait sous un angle particulier puisqu'il s'agira notamment de s'interroger sur la vitalité de son usage et sur ce que cela implique en termes d'enseignement. Nous commencerons par la décrire formellement, en précisant l'orientation théorique qui semble la plus appropriée pour l'analyser, ce qui permettra d'envisager des pistes possibles pour l'enseigner.

De fait, cette contribution peut s'inscrire dans une problématique plus large concernant la place à accorder, dans le cadre de l'enseignement/apprentissage des LVR, à des notions ou des structures syntaxiques peu connues de la part des élèves et à leurs modalités d'enseignement.

\section{La réduplication en miroir en créole analyse et description}

On note que la réduplication est un phénomène courant lorsque l'on se place dans une optique de linguistique générale; phénomène diversement utilisé selon les langues, qui peuvent lui associer des valeurs diverses ${ }^{3}$. On soulignera, à la suite notamment de Parkwall (2003) et de Parkwall et Bakker (2005), que la réduplication est un procédé caractéristique des langues

\footnotetext{
${ }^{1}$ Les astérisques renvoient aux termes qui sont présentés dans le glossaire.

${ }^{2}$ Les exemples, présentés sur quatre lignes, comportent outre la graphie, la transcription phonétique en seconde ligne, le signifié de chacune des unités, en troisième ligne et enfin, la traduction de l'énoncé, en quatrième ligne. Les abréviations utilisées dans le cadre de cet article sont les suivantes : $1:$ première personne ; $2:$ deuxième personne ; 3 : troisième personne $; \mathrm{SG}:$ singulier ; PL : pluriel (1SG fait donc référence au pronom personnel $11^{\text {ère }}$ personne $\mathrm{du}$ singulier, $2 \mathrm{SG}$ au pronom personnel $2^{\text {ème }}$ personne $\mathrm{du}$ singulier, etc.) ; ACCOMP.: accompli* (correspondant à un signifiant ZERO noté $\emptyset$ ) ; INACC. : inaccompli* ; IRR. : irréalisé* ; DEF. : défini ; INDEF. : indéfini ; REL. : relateur*; FOC. : focalisateur. Par ailleurs, les exemples présentés dans le cadre de cet article sont en créole guadeloupéen. Pour le créoles martiniquais, la structure est similaire avec des variations en ce qui concerne la forme du pronom; ces variations, liées au contexte phonétique et donc sans lien avec la structure elle-même, s'expliquent par le fait qu'il n'existe pas de relateur en créole martiniquais entre le pronom personnel et l'unité qui le précède dans de tels cas. Ainsi, à l'énoncé (1) correspond en créole martiniquais : Kité'y alé alé'y.

${ }^{3}$ Cf. par exemple la valeur diminutive associée à la réduplication des unités verbales dans les créoles de l'Océan Indien. Exemple en créole réunionnais : Zot la danss-danss séga. \{3pl. acc danser FC danser FC séga « Ils ont un peu dansé le séga. » (Staudacher-Valliamée, 2007 ; comme le précise l'auteur dans ce créole «90\% des thèmes verbaux connaissent une double forme, longue et brève à l'accompli ». La mention FC dans l'exemple indique qu'il s'agit de la forme courte de l'unité verbale). Se référer également à Bakker (2003).
} 
créoles. En ce qui concerne plus précisément la réduplication de l'unité verbale, en créoles martiniquais et guadeloupéen, on observe que ce procédé confère une valeur signifiée à l'énoncé, distincte du sens véhiculé lorsque le verbal ${ }^{4}$ n'est pas rédupliqué. La réduplication est utilisée majoritairement ${ }^{5}$ dans les deux cadres suivants :

- l'intensification: la réduplication permet alors de rendre compte d'une valeur augmentative du procès.

(3)i atann atann jis jou wouvè

[ i $\varnothing$ atãn atãn zis zu wuve ]

3SG ACC attendre attendre jusque jour ouvrir

« Il a attendu longuement, jusqu'à ce que le jour se lève.

(litt. « Il a attendu attendu jusqu'à ce que le jour ouvre)»

(4)tout lè ou ka wè’y, i ka grigné grigné.

[ tut le u ka we j i ka grine gyine ] tout heure 2SG INACC voir 3SG 3SG INACC grimacer grimacer «A chaque fois qu'on le voit, il n'arrête pas de grimacer. »

A travers ces exemples, ce que l'on peut constater c'est que la réduplication du verbal permet de souligner la fréquence, l'ampleur, la durée étendue du procès auquel il est fait référence. Quelle que soit la nature du procès, il y a donc notation d'une certaine amplification. La valeur signifiée globale de l'énoncé est donc différente d'un énoncé sans réduplication de l'unité verbale.

(5)i atann jis jou wouvè

[ i $\varnothing$ atãn zis $\quad$ zu wuve ]

3SG ACC attendre jusque jour ouvrir

«Il a attendu jusqu'à ce que le jour se lève. »

(6)lè i ka souri, i ka grigné.

[ le i ka suri i ka grije]

quand 3SG INACC sourire 3SG INACC grimacer

«Quand il sourit, il grimace. »

- la mise en valeur : la réduplication du verbal, dont l'une des occurrences* est antéposée à l'unité sujet, permet de mettre l'accent sur le procès. L'unité ainsi mise en valeur est atann dans l'exemple (7) et grigné dans l'exemple (8).

(7)(sé) atann i atann

[(se) atãn $\quad$ i $\quad \varnothing \quad$ atãn $]$

FOC. attendre 3SG ACC attendre

« Il a vraiment attendu. » (Litt. : « C'est attendre, qu'il a attendu. »)

\footnotetext{
${ }^{4}$ Le terme de verbal est utilisé pour designer les unités qui assument de façon privilégiée le rôle de noyau d'énoncé, sans qu'il s'agisse de leur vocation exclusive. Le terme de verbe est donc écarté dans la mesure où ces unités ne partagent pas toutes les caractéristiques du verbe, tel que défini en linguistique générale. Cf. Beatrice Jeannot-Fourcaud (2003).

${ }^{5}$ La réduplication du verbal peut être utilisée dans d'autres cadres (expression du rapport temporel ou du rapport de causalité entre deux procès, expression de la concession). Cf. notamment Damoiseau (1999).
} 
(8)(sé) grigné i ka grigné.

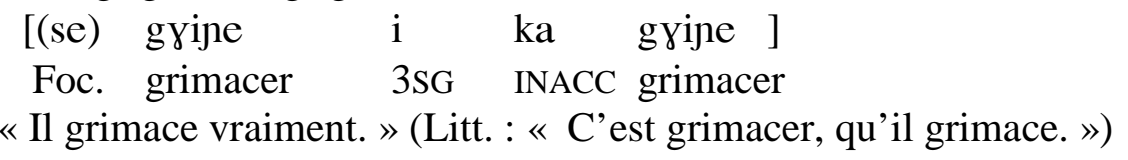

Dans ce type de réduplication, le focalisateur* [se] est facultatif ; c'est le fait de rédupliquer l'unité verbale en antéposant l'une de ses occurrences qui permet la mise en valeur du procès*6.

Dans le cadre qui nous intéresse ici, on mentionnera un autre type de réduplication, celle mise en scène dans les exemples (1) et (2), à savoir la réduplication du verbal qui s'accompagne de la postposition d'une occurrence du pronom personnel et qui pose quelques interrogations en termes de description et d'analyse syntaxique. Comme dans les cas cités précédemment, la réduplication confère également ici une valeur sémantique spécifique à l'énoncé, comme on peut le voir dans l'exemple (11) par contraste avec les exemples (9) et (10).

$\begin{array}{llll}\text { (9)Misyé-la } & \text { ka } & \text { souri } \\ {[\text { misje }} & \text { la } & \text { ka } & \text { suri ] } \\ \text { monsieur } & \text { DEF. } & \text { INACC. sourire } \\ \text { «Le monsieur sourit. » / « Il sourit. » }\end{array}$

(10) Gadé misyé-la ka souri souri. I dwèt ka fè lafèt épi mwen.

$\begin{array}{lllllll}\text { [ gade } & \text { misje } & \text { la } & \text { ka } & \text { suri } & \text { suri } & \text { i } \\ \text { regarder } & \text { monsieur } & \text { DEF. Inacc. } & \text { sourire } & \text { sourire } & \text { 3sG } \\ \text { dwet } & \text { ka } & \text { felafetepi } & \text { mw } \tilde{\varepsilon} & \text { ] } & \\ \text { PROB. } & \text { INACC. } & \text { se moquer de } & 1 \mathrm{SG} & & \end{array}$

« Regarde le, qui n'arrête pas de sourire. Il doit se moquer de moi. »

(11)Lésé yo souri souri'a-yo. Sa pa ka fè ayen

\begin{tabular}{|c|c|c|c|c|}
\hline [lese & suyi & suyi & $\mathrm{a}$ & jo \\
\hline $\begin{array}{l}\text { laisser } \\
\text { pa }\end{array}$ & $\begin{array}{l}\text { 3PL sourire } \\
\mathrm{ka}\end{array}$ & $\begin{array}{c}\text { sourire } \\
\text { a } \tilde{\tilde{e}}\end{array}$ & REL & $3 \mathrm{PL}$ \\
\hline
\end{tabular}

NEG. INACC. faire rien

«Laisse les sourire autant qu'ils veulent, cela ne fait rien. »

L'exemple (12), par contraste avec les énoncés (5) et (7), nous permet également de voir la valeur sémantique véhiculée par cette structure.

(12)I atann atann'a-y men i té sav nou pé té ké vini.

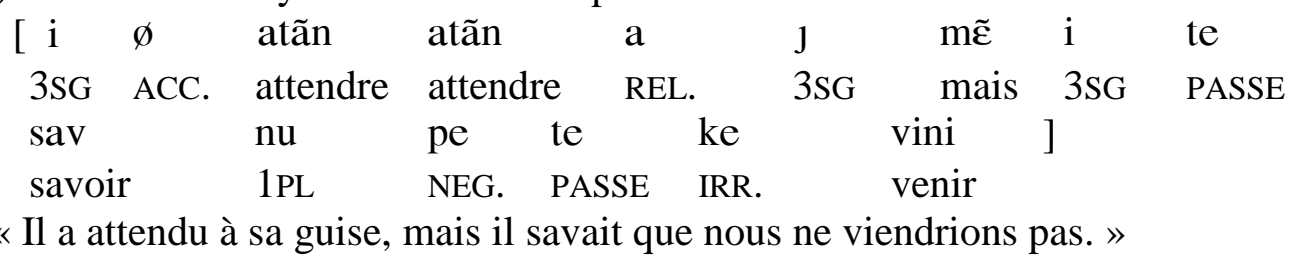

\footnotetext{
${ }^{6}$ On peut noter que ce type de structure se retrouve dans une certaine mesure dans le français régional antillais, où l'on peut entendre des phrases comme «C'est dormir qu'il dort». cf. l'article d'André Thibaud (2010) portant sur cette structure en français régional.
} 
La dimension sémantique véhiculée dans ce type d'exemple est explorée plus avant dans le cadre de la description de la structure concernée (cf. infra).

\subsection{Caractéristiques de la réduplication en miroir $^{7}$}

La structure de réduplication en miroir, telle que présentée dans les exemples (1), (2), (11) et (12), se caractérise par la réduplication de l'unité verbale qui apparaît suivie d'une occurrence du pronom personnel. En ce qui concerne le pronom personnel qui apparaît ainsi à la suite du segment formé par les deux occurrences du verbal, il peut s'agir soit de la réduplication du personnel en fonction sujet (ex. 13) soit tout simplement du « rappel » du référent de l'unité en fonction sujet (ex. 14). La seconde occurrence du personnel est donc co-référente de l'unité en fonction sujet.

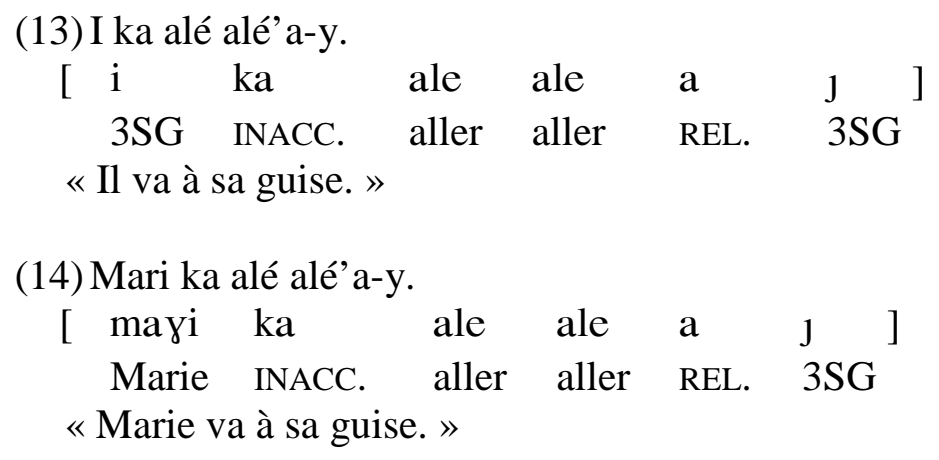

On observe que l'occurrence postposée du pronom personnel fait nécessairement écho au référent de l'unité en fonction sujet. Un énoncé tel que celui présenté dans l'exemple (15) est donc agrammatical :

$$
\begin{aligned}
& \text { (15) *I ka alé alé an-mwen. }{ }^{8}
\end{aligned}
$$

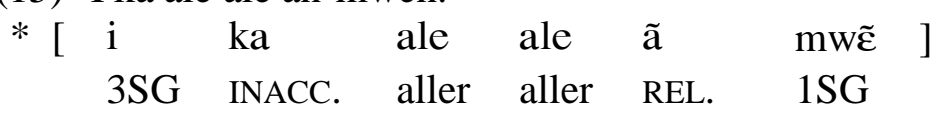

Par ailleurs, on constate que cette structure ne concerne pas tous les types de verbaux, certains d'entre eux sont totalement exclus de ce type de structuration, et ce, qu'ils soient transitifs ou intransitifs. Les observations montrent que l'on peut mettre en relation l'incompatibilité entre certains verbaux et cette structure, d'une part, et la valeur sémantique de ces verbaux, d'autre part.

Il apparaît en effet que les verbaux qui ne peuvent entrer dans ce type de construction sont ceux dont le participant ne peut avoir de contrôle sur le procès. Ainsi, un énoncé tel que l'énoncé (16) avec l'unité verbale vwè « voir » n'est pas grammatical, alors que l'énoncé (17) avec le verbal gadé « regarder » l'est :

$$
\begin{aligned}
& \text { (16) * I ka vwè vwè'a-y. }
\end{aligned}
$$

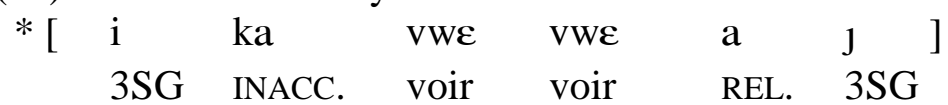

\footnotetext{
${ }^{7}$ Pour des données supplémentaires d'un point de vue descriptif et théorique, cf. Béatrice Jeannot-Fourcaud (2011) et Béatrice Jeannot-Fourcaud (2012).

${ }^{8}$ Le signe * placé avant un énoncé indique son agrammaticalité.
} 
(17)I ka gadé gadé'a-y.

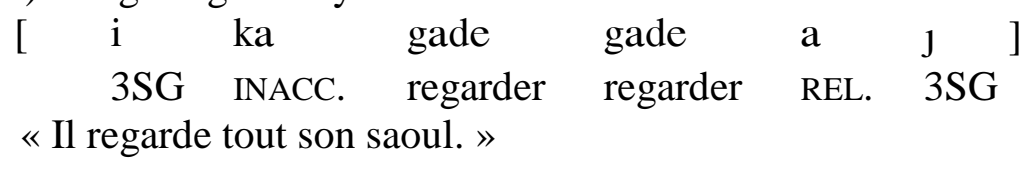

On conçoit effectivement, que le procès «voir » n'est pas dépendant de la volonté de l'agent, contraint de voir ce qui se trouve sous ses yeux, alors qu'à l'inverse, le fait de regarder peut impliquer un certain contrôle ou une certaine volonté de la part de celui qui fait l'action.

De même les unités verbales rendant compte d'une sensation physique, comme dans les exemples (18) et (19) ne peuvent non plus entrer dans ce type de structure.

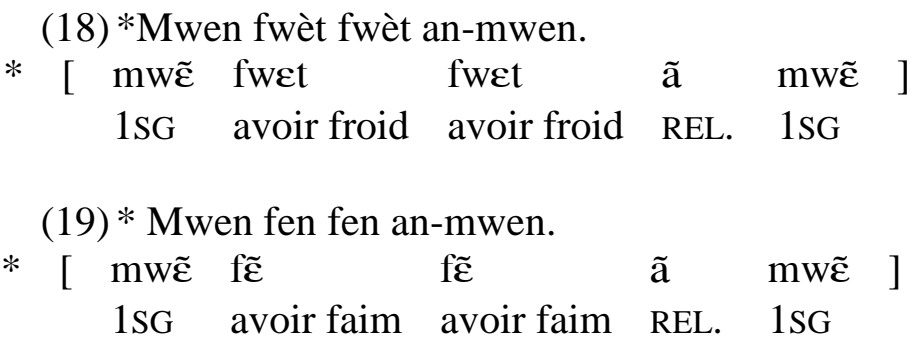

Pour qu'une unité verbale soit compatible avec ce type de structure, il semble donc que le sens qu'il véhicule doive induire un minimum de prise de contrôle de l'agent sur le procès auquel il est référé, c'est-à-dire que l'agent puisse avoir un certain « contrôle » sur le procès.

\subsection{Analyse théorique ${ }^{9}$}

Ces différentes observations m'ont amenée à considérer la définition théorique de la voix, telle qu'énoncée par Myriam Klaiman (1991), à travers les notions de basic voice et de derived voice. Dans cette acception, tout comme en grammaire traditionnelle, la notion de voix associe la modification de la structure d'une phrase à une modification sur le plan sémantique des participants engagés dans le procès ${ }^{10}$. Dans la perspective proposée par Klaiman, cependant, la voix est plus spécifiquement conçue comme une modification du rapport du participant en fonction sujet au procès. Dans le cadre de la basic voice, la focalisation est mise sur la relation établie entre le procès désigné par le verbe et le participant en fonction sujet. A l'inverse de ce que Klaiman nomme la derived voice, la basic voice ou voix typique (pour la traduction, cf. Lazard 2001: 66), se caractériserait donc ici par la permanence des fonctions lors du passage de l'énoncé non marqué (I ka gadé, exemple 20) à

\footnotetext{
${ }^{9}$ Pour des propositions d'analyse différente, cf. Jean Bernabé (1983) et Jean Bernabé (2003) notamment. Cf. également Béatrice Jeannot-Fourcaud (2011) pour une discussion concernant le point de vue impliquant la notion d'objet ou celle de complément interne.

${ }^{10}$ Le terme de participant réfère au plan sémantique. Ainsi, une unité qui assume la fonction sujet peut remplir différents rôles sémantiques selon la phrase (agent, patient, expérient, source...). Le rôle de chaque unité au sein d'un énoncé peut donc être caractérisé sous deux plans différents : du point de vue de la fonction syntaxique qu'elle assume et du point de vue de son rôle sémantique. Par exemple, en français, dans une phrase telle que $L e$ chat mange la souris, chat assume la fonction sujet et le rôle sémantique d'agent (celui qui fait l'action) alors que souris assume la fonction objet et le rôle sémantique de patient (celui qui subit l'action). Telle quelle est enseignée traditionnellement en France, la transformation de voix implique qu'à une différence de construction (forme active $v s$ voix passive), corresponde une modification des fonctions syntaxiques assumées par les unités référant aux participants au procès. Ainsi, à l'énoncé actif cité ci-dessus Le chat mange la souris, correspond l'énoncé passif La souris est mangée par le chat, dans lequel, souris, tout en conservant le rôle sémantique de patient devient sujet du verbe, alors que parallèlement, chat, tout en demeurant, sur le plan sémantique, agent, assume la fonction de complément d'agent.
} 
l'énoncé marqué par la voix (I ka gadé gadéa-y; exemple 21) mais également par une modification du degré d'implication du participant en fonction sujet ${ }^{11}$.

(20) I ka gadé.

[ $\mathrm{i}$ ka gade ]

3SG INACC. regarder

« Il regarde. »

(21)I ka gadé gadé a-y.

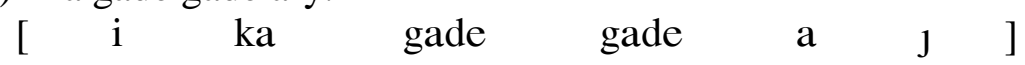

3SG INACC. regarder regarder REL. 3SG

«Il regarde tant qu'il peut. »

Ainsi dans l'énoncé (20), le référent de [i] est l'agent, qui accomplit le procès de « regarder »; dans l'énoncé (21), on relève une implication supplémentaire, que j'ai mise en relation avec la notion de contrôle potentiel que peut avoir un agent sur le procès; ce qui semble confirmé par la distribution des verbaux en fonction de leurs capacité ou non à entrer dans ce type de structure et leur valeur sémantique, comme mentionné précédemment. On peut donc postuler que l'effet de sens véhiculé par cette structure consiste en une augmentation du degré d'implication et de contrôle de l'agent sur le procès.

\section{Une structure en régression?}

Les observations des usages, confirmées par une enquête menée en Guadeloupe auprès d'une population d'élèves de cycle $3^{12}$, tendent à indiquer qu'il s'agit là d'une structure en régression, ce que l'on peut constater à deux niveaux. Tout d'abord sur le plan quantitatif on peut noter une baisse de sa fréquence d'emploi (3.2). On peut également mesurer une évolution de la valeur sémantique qui lui est attribuée (3.3).

\subsection{Méthodologie de recueil de données}

Les données concernant la structure de réduplication à l'étude ont été recueillies dans le cadre d'une enquête menée auprès d'une population de cinquante-et-un élèves de cycle 3 (une classe de CE2/CM1 et une classe de CM1/CM2) d'une école publique des Abymes. Les enfants sondés présentent des profils langagiers différents, en fonction du nombre de langues qu'ils parlent notamment, mais on note malgré tout, au sein de la population à l'étude, une convergence fondamentale, puisqu'ils sont tous bilingues français-créole, même si pour l'un d'entre eux la compétence en créole est une compétence "passive ». En ce qui concerne le créole, plus précisément, tous les élèves déclarent comprendre le créole guadeloupéen, certains comprenant en plus un autre créole $^{13}$ (haïtien et martiniquais, principalement mais également le créole de la Dominique et le créole guyanais). Excepté un élève qui déclare ne parler aucun créole, tous parlent au moins un créole, voire deux (9 élèves) ou trois (1 élève).

\footnotetext{
${ }^{11}$ Contrairement à ce que l'on a vu pour la voix passive en français, dans le cadre de la basic voice, seul le participant en fonction sujet est impliqué.

${ }^{12}$ Enquête menée par Frédéric Anciaux et l'auteure en mai 2011.

${ }^{13}$ Tous de la zone américano-atlantique.
} 
Parmi ceux qui déclarent ne parler qu'un créole (39 élèves), 35 citent le créole guadeloupéen, et les autres un des autres créoles antillais ${ }^{14}$.

Nous avons testé deux phrases comportant la structure de réduplication en miroir, dans le cadre d'une passation de groupe effectuée en mai 2011. Chaque phrase était lue par l'enquêteur puis les consignes énoncées; les élèves avaient la possibilité d'interroger l'enquêteur en cas de non-compréhension des consignes ou pour des demandes de précision.

Les deux phrases testées étaient les suivantes ${ }^{15}$ :

Nou té ka domi domi an-nou lè yo sonen.

Lésé yo souri souri a-yo, sa pa ka fè ayen.

Les traductions n'étaient pas proposées aux élèves ; ceux-ci devaient se positionner sur trois plans (cf. annexe). Tout d'abord, ils devaient dire si la phrase qui leur avait été lue avait un sens pour eux ; le cas échéant, il leur était demandé d'en indiquer le sens ; et enfin, de préciser s'ils pourraient eux-mêmes employer cette phrase.

Cette méthode présentait l'avantage d'explorer non seulement le fait que cette structure soit perçue comme porteuse de sens, de mesurer l'écart entre le sens attribué à cette structure par les élèves et le sens basilectal qui y est associé, et également de prendre connaissance de leurs pratiques déclarées quant à son usage éventuel.

\subsection{Baisse d'emploi}

Les résultats des tests menés auprès de la population d'élèves indiquent clairement une baisse d'emploi de cette structure, mesurée à travers les mécanismes de réception (compréhension d'une phrase à structure de réduplication à miroir) et de production (pratique déclarée quant à l'utilisation possible d'une phrase à réduplication en miroir). Les résultats exposés ci-dessous concernent l'exemple : nou té ka domi domi an nou, présentée aux élèves sans traduction, selon le protocole exposé en annexe.

En ce qui concerne l'aspect lié à la réception tout d'abord, on observe un déficit de reconnaissance de cette structure chez une partie d'entre eux, comme nous pouvons le voir avec la figure 1. En effet, à la question : "Cette phrase a-t-elle un sens pour toi ? », dix élèves ont répondu par la négative. Il est également intéressant de voir que quatre d'entre eux n'ont pas répondu à la question.

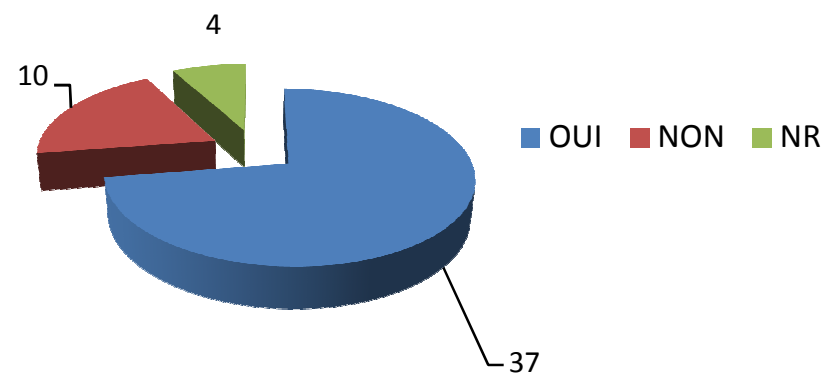

Figure 1 : Compréhension d'une phrase à structure de réduplication en miroir

\footnotetext{
${ }^{14}$ Cette précision est importante puisque cette structure ne semble pas validée en créole guyanais. Concernant l'élève qui déclare ne parler aucun créole, nous avons fait le choix de ne pas l'exclure de l'échantillon, pour la variable à l'étude, dans la mesure où cet élève déclare comprendre le créole guadeloupéen.

${ }^{15}$ Cf. pour la segmentation et la traduction de ces phrases, les exemples (2) et (11).
} 
On constate cependant que si près de $20 \%$ des élèves déclarent ne pas comprendre une telle phrase, une majorité d'entre eux déclarent au contraire la comprendre ${ }^{16}$. On pourrait par conséquent en déduire que le fait que cette structure soit comprise par une majorité des élèves en fait une structure dont l'usage est avéré dans la population guadeloupéenne.

Cela étant si l'on considère les résultats à la question posée en relation avec le taux de production éventuelle de cette phrase (cf. figure 2), on constate que le nombre d'élèves déclarant pouvoir produire une phrase de ce type (vingt-sept élèves) baisse considérablement au regard du taux de ceux qui déclarent la comprendre (trente-sept élèves), de même que le taux de non-réponse augmente de façon significative.

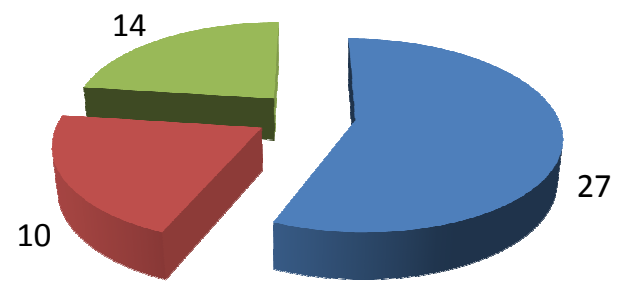

oui $\square$ non $\square \mathrm{nr}$

\section{Figure 2 : Nombre d'élèves déclarant qu'ils pourraient produire cette phrase}

Or, si l'on postule que l'on peut mesurer la vitalité d'une variante linguistique en mesurant d'une part l'écart qu'il y a entre production et réception ${ }^{17}$, ces résultats semblent confirmer l'hypothèse selon laquelle cette structure est en régression. Comme nous allons le voir, d'autres données corroborent ces premières constatations.

\subsection{Désémantisation}

Comme précisé, une majorité des élèves déclarent comprendre cette phrase. Le taux de réception est donc relativement élevé et l'on pourrait donc en déduire que même si seulement un peu plus de la moitié d'entre eux se déclarent susceptible de produire une phrase de ce type, cette structure conserve une assez bonne vitalité. Pourtant ces résultats, en termes de réception, doivent être relativisés du fait que parmi les élèves qui affirment comprendre la phrase testée, les interprétations sémantiques divergent totalement puisque l'on se trouve face à plusieurs interprétations concurrentes. Il s'agit également, selon nous, d'un indicateur qui confirme que cette structure est en régression, tout au moins du point de vue de ce qu'elle signifie. En effet, le manque de stabilité au niveau du sens véhiculé par une unité lexicale ou par une structure syntaxique nous renseigne systématiquement sur un processus évolutif en cours. En l'occurrence, on peut faire deux hypothèses : soit, la structure en elle-même se maintient mais son sens évolue vers un sens différent (tout comme une unité lexicale qui, tout en restant dans le lexique d'une langue, peut voir son sens se modifier au fil du temps), soit la structure en elle-même est très peu utilisée ce qui explique de fait que les élèves, l'entendant et l'utilisant peu, l'associent à des sens divers.

\footnotetext{
${ }^{16}$ Nous verrons cependant que le sens attribué à cette phrase par ceux qui déclarent la comprendre, est variable.

${ }^{17}$ En effet, lorsque deux variantes linguistiques sont en concurrence, l'abandon de l'une d'entre elle se traduit au préalable par une baisse de son emploi même si elle demeure pendant un temps reconnue. Bien évidemment, le facteur de l'âge est prépondérant dans le cadre de la mesure de la vitalité d'une variante dans la mesure où la dynamique d'une évolution en cours se mesure par l'écart entre l'usage des jeunes générations et des générations plus anciennes.
} 
Au regard des données présentées en ce qui concerne la baisse d'emploi (cf. 3.2), cette seconde hypothèse parait plausible mais également confirmée par l'analyse qui suit. Comme mentionné précédemment, l'interprétation basilectale d'une phrase comportant la structure de réduplication en miroir, telle que nou té ka domi domi nou est «Nous dormions tout notre saoul/ à notre guise ». Or, seuls à peine quatre élèves ont proposé cette traduction. Parmi les réponses des autres élèves, on constate que les équivalences sémantiques données s'en écartent. On trouve ainsi les interprétations concurrentes suivantes, présentées ci-dessous brièvement et illustrées par la figure 3 .

- «Nous dormions » (quinze élèves)

- «Nous dormions beaucoup » (deux élèves)

- « Nous dormions notre sommeil/notre somme » (un élève)

- Autres : Il s'agit de propositions idiosyncrasiques, par exemples « Nous dormions sur nous »; « nous dormions en créole », etc.

Si l'on analyse ces différentes propositions, on peut faire les constats suivants :

- «Nous dormions » : cette interprétation, la plus courante parmi les élèves, revient à réduire le sens de la phrase à celui véhiculé par une phrase sans aucune réduplication, (Nou té ka dòmi). Cette variante est nommée dans la figure $3:<$ réduction au sens sans réduplication>.

- «Nous dormions beaucoup »: quelques élèves interprètent cette phrase comme une phrase à réduplication augmentative (cf. supra), c'est-à-dire comme si elle comportait simplement la réduplication de l'unité verbale (Nou té ka dòmi dòmi.). Cette variante est nommée dans la figure $3:$ : réduction au sens avec réduplication simple〉.

- «Nous dormions notre sommeil/notre somme»: cette interprétation, consistant à considérer la seconde unité verbale comme un nom déterminé par un pronom personnel en fonction génitive ( «son somme ou son sommeil ») a finalement été très peu retenue alors que l'une des hypothèses initiales de ce travail était précisément que la désémantisation se faisait principalement en faveur de cette variante, nommée dans la figure 3 : <interprétation nominale>.
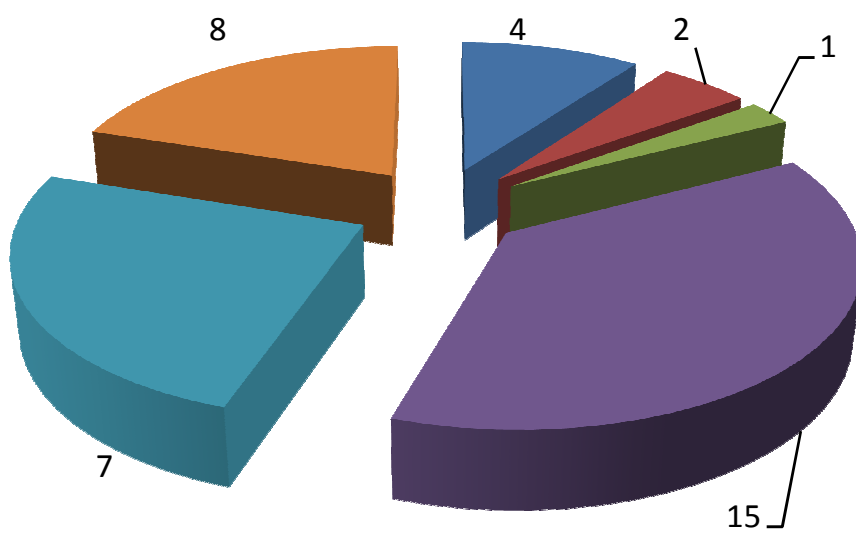

- Interprétation basi : 1

Réduction rédupl.simple : 2

- Interprétation nominale : 3

Réduction au sens sans rédupl. : 4

NSP \& NR : 5

autre : 6

Figure 3 : Interprétation sémantique de Nou té ka domi domi an nou

Parmi les trente-sept élèves ayant déclaré comprendre cette phrase, seuls quatre lui attribuent la signification basilectale. Il est, par ailleurs, notable que les deux scores les plus élevés, après les réponses concernant une réduction au sens sans réduplication, sont le score de nonréponse ou NSP ou d'autres interprétations ; ce qui confirme l'instabilité de cette structure (Houdebine, 1996) au sein de la population à l'étude. 
Quoi qu'il en soit, ces premiers résultats nous amènent à déduire que cette structure n'est plus d'un usage généralisé en Guadeloupe et qu'elle est même en nette régression. Il s'agit d'un constat général, qui masque sans nul doute des pratiques divergentes. La fréquence d'utilisation de cette structure ainsi que l'interprétation qui y est associée varient nécessairement selon les locuteurs en fonction notamment du milieu sociolinguistique dans lequel ils évoluent et donc des pratiques familiales et sociales. On postule aussi que l'âge est un facteur prépondérant dans la distribution sociolinguistique de cette variable.

D'ailleurs, le fait que son usage apparaisse en régression dans une population jeune nous incite à nous interroger sur son avenir. On notera cependant que les données présentées concernent un échantillon restreint (51 élèves). De ce fait, même si les observations in vivo semblent confirmer cette tendance, il conviendrait bien entendu de mener une étude au sein d'une population plus vaste afin de voir, d'une part, si ces résultats sont validés ou non au sein de cette classe d'âge en Guadeloupe et d'autre part, s'ils peuvent être généralisés. En outre, il serait nécessaire de mettre en relation ces données avec les variables d'ordre social ce qui permettrait une analyse plus fine de la distribution de cette variable au sein de la population. Il y a lieu de se demander, à ce stade de l'analyse, s'il est parfaitement adéquat d'utiliser le terme de désémantisation au lieu d'évoquer simplement un processus d'évolution sémantique, phénomène courant dans le processus d'évolution de toute langue.

Cependant, dans ce cas, il peut sembler approprié de considérer que l'on a réellement affaire à un phénomène de désémantisation dans la mesure où la valeur sémantique n'est pas transférée vers une autre valeur qui lui serait spécifique. Il y a une perte sémantique dans la mesure où le sens de cette structure semble se confondre majoritairement, en tout cas dans la population à l'étude, avec la valeur sémantique d'une phrase sans réduplication. Ainsi, pour près de $30 \%$ des élèves, nou té ka domi domi an-nou est interprété tout simplement de la même façon que nou té ka domi «Nous dormions. » L'observation des pratiques des élèves et plus particulièrement des interprétations qu'ils font de la structure peuvent nous donner des pistes intéressantes relativement à son enseignement.

\section{Des pistes pour son enseignement ${ }^{18}$}

Compte-tenu de la description faite précédemment, un enseignement en grammaire du groupe verbal pourrait être adapté. En effet, si l'on adhère à l'analyse effectuée, il s'agit donc de relations syntaxiques au sein du groupe verbal, qui peuvent être traitées dans le cadre de la détermination d'un verbal par une unité de temps, d'aspect ou de voix. Ainsi au même titre que té et $k a$ sont analysés dans la phrase Nou té ka dòmi «Nous dormions », comme des déterminants grammaticaux de l'unité verbale, respectivement, unité de temps PASSE et unités d'aspect ACCOMPLI (cf. représentation qui suit),

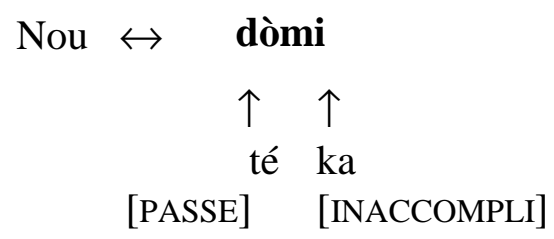

\footnotetext{
${ }^{18}$ Une sensibilisation pourrait être faite au primaire présentant les différentes structures de réduplication (cf. supra). Cependant, les propositions de pistes qui suivent sont plutôt destinées à un public de collégiens dans le cadre d'un enseignement des LVR.
} 
On peut représenter la réduplication du verbal et la co-référence du pronom sur le même modèle.

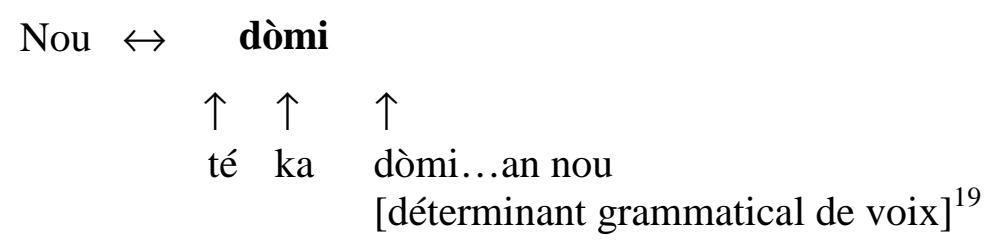

Cela étant, on note que la notion de voix peut être difficile à acquérir, du fait des différentes notions qu'elle suppose et qui doivent donc être préalablement acquises, avant de l'aborder. On peut de fait s'interroger sur le risque de confusion que pourrait engendrer le fait d'introduire deux points de vue différents (le point de vue traditionnel qui sert de cadre pour enseigner le passif en français ou en anglais par exemple, et le point de vue de Klaiman) auprès des élèves.

On peut également choisir de présenter de façon systématique les structures de réduplication, dont la réduplication en miroir, sans entrer dans l'analyse théorique qui les sous-tendent et les catégoriser, tout en sachant cependant que les différents types de prédication présentés relèvent de domaines différents :

- La réduplication simple (exemples 3, 4 et 10) relève du lexique, puisqu'elle permet la création d'unités lexicales, de «mots composés».

- La réduplication avec antéposition de l'une des occurrences du verbal (exemples 7 et 8 ) correspond à un processus de mise en valeur.

- La réduplication en miroir est à traiter dans le cadre de la détermination des verbaux.

Les principaux points qu'il nous semble important de garder à l'esprit, toutefois, et qu'il convient donc de bien souligner auprès des élèves sont les suivants :

- Le second verbal ne doit pas être traité comme fonction nominale, c'est-à-dire comme une fonction que le nom peut assumer. En effet, on considère qu'il ne peut en aucun cas s'agir d'une fonction nominale puisque comme nous l'avons mentionné, cette structure est valide également pour les verbaux intransitifs. En outre, l'unité rédupliquée ne peut être considérée comme un nom, mais bien comme un verbal. Ceci est confirmé par le fait que cette unité ne peut être déterminée par un déterminant du nom, de type article. ${ }^{20}$

- Cette structure diffère donc d'une phrase canonique comportant sujet+verbal+objet, dans lequel l'objet est représenté par un nom homophone du verbal, tel que dans l'exemple (22).

(22) I ka bwè bwè $a-y$.

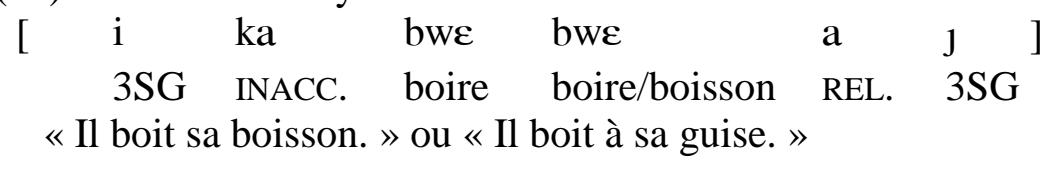

L'interprétation première que cette phrase évoque on l'a vu met en scène un nom déterminé par un pronom personnel en fonction génitive, ce qui marque donc la possession : « Il boit sa

\footnotetext{
${ }^{19}$ J'ai fait par ailleurs différentes propositions pour nommer cette unité de voix : voix ascendante (JeannotFourcaud, 2003 et 2012); voix de contrôle ou controlled voice (Jeannot-Fourcaud, 2012)

${ }^{20}$ Cf. Jeannot-Fourcaud (2011).
} 
boisson ». Cependant, dans un contexte spécifique, tel que dans (23), le sens associé à la réduplication en miroir peut être retenu.

(23) Lésé'y bwè bwè a-y. I ké wè lè I ké malad.

$\begin{array}{lllllll}\text { [ lese } & \mathrm{J} & \text { bw } \varepsilon & \text { bwe } & \text { a } & \text { J } & \text { i } \\ \text { laisser } & 3 S G & \text { boire } & \text { boire/boisson } & \text { REL. } & \text { 3SG } & \text { 3SG } \\ \text { ke } & \text { we } & \text { le } & \text { i } & \text { ke } & \text { malad } & \text { ] } \\ \text { IRREAL. } & \text { voir } & \text { comme } & \text { 3SG } & \text { IRREAL. } & \text { 3SG } & \\ \text { « Laisse le boire à sa guise. Il verra quand il sera malade. » }\end{array}$

Comme on le voit, une phrase de ce type est susceptible de recevoir les deux types d'interprétations ; cette double interprétation est rendue possible par l'homophonie entre nom et unité verbale, dans le cas présenté. Dans cette optique, il peut être judicieux de différer ce type d'énoncé en présentant d'abord des énoncés non équivoques.

- Il convient également de faire observer qu'il s'agit d'une structure productive en créole puisque l'on peut l'utiliser pour un grand nombre d'unités verbales.

- Par ailleurs, il est fondamental de montrer que le caractère grammatical de cette structure dépend du contrôle que l'agent peut avoir sur le procès dénoté par le verbal. Dans cet objectif, on peut faire prendre conscience de la notion de contrôle en évoquant les verbaux de sensation par contraste avec les verbaux référant à des actions.

\section{Conclusion}

Bien entendu ces pistes ne sont envisageables que si l'on conçoit la légitimité d'enseigner une telle structure. Deux perspectives antinomiques peuvent être évoquées. D'une part, on peut considérer que cette évolution, qui pourrait aboutir à terme à la disparition de cette structure, est le résultat «normal » du processus d'évolution que connait toute langue en usage. On sait en effet que toute langue vit parce qu'elle évolue, parce qu'elle s'adapte aux nouvelles réalités à décrire et qu'elle se plie aux exigences d'économie linguistique, et donc, en définitive, qu'elle se plie à l'usage qu'en font les locuteurs. L'évolution peut-être lexicale, syntaxique, phonétique ou encore sémantique, comme dans le cas qui nous intéresse ici. Une éventuelle disparition de cette structure induirait ainsi que la langue et donc les locuteurs n'en ont plus le besoin.

Cela étant, une autre perspective peut être évoquée. Pour rappel, nous avons vu précédemment que la réduplication est considérée comme particulièrement caractéristique des créoles. A ce titre, le processus de désémantisation décrit précédemment, semble remettre en cause cette particularité du créole. En effet, réduire le sens d'une phrase mettant en scène cette structure au sens d'une phrase sans réduplication aucune, revient à rendre caduque le processus même de réduplication, qui à terme peut n'avoir aucune pertinence significative. En outre, et au-delà de la simple défense d'une structure syntaxique, l'étude de ce type de structure semble particulièrement fécond, dans le cadre du développement des compétences métalinguistiques, de réflexion sur la/les langue(s). Profiter du potentiel bilingue des élèves pour leur montrer les divergences de fonctionnement entre langues, quelles qu'elles soient, ne peut être que bénéfique. Dans la mesure où français et le créole présentent sur ce plan, comme dans d'autres domaines, des divergences très nettes, l'étude de ce point de syntaxe particulier permettrait notamment de montrer que les langues ne s'organisent pas de la même manière et que chacune peut utiliser des procédés qui lui sont totalement spécifiques, comme la 
réduplication pour le créole. En ce qui concerne la légitimité et les modalités de son enseignement, la question reste ouverte.

\section{Références bibliographiques}

Bakker, P. (2003). Reduplication in Maurtian Creole with notes on reduplication in Reunion Creole. Dans S. Kouwenberg (dir.), Twice as meaningful. Reduplication in Pidgins, Creoles and other contact languages (p. 211-218). Westminster Creolistic Series 8.

Bakker, P. et Parkwall, M. (2005). Reduplication in Pidgins and Creoles. Dans B. Hurch (dir.), Studies on reduplication (p. 511-531). Mouton de Gruyter.

Bernabé, J. (2003). Précis de syntaxe créole. Guadeloupe, Martinique, Guyane, Paris et Réunion : Ibis Rouge Editions.

Bernabé, J. (1983). Fondal-Natal, Grammaire approchée des créoles guadeloupéens et martiniquais. Paris : L'Harmattan.

Cresseils, D. (1995). Eléments de syntaxe générale. Paris : PUF.

Cresseils, D. (2006). Syntaxe générale, une introduction typologique. Paris: Hermès Lavoisier.

Damoiseau, R. (1999). Eléments de grammaire comparée Français, Créole. Petit Bourg : Ibis Rouge Editions.

Foley, W.A. et Van Valin, R. D. (1984). Functionnal syntax and universal grammar. Cambridge : Cambridge University Press.

Givón, T. (1994) The pragmatic of de-transitive voice : Functional and typological aspects of inversion. Dans T. Givon (dir.), Voice and inversion (p. 1-46). Amsterdam/Philadelphia : Benjamins.

Houdebine, A-M. (dir.) (1996). Travaux de linguistique, Imaginaire linguistique, 7, Université d'Angers.

Jeannot-Fourcaud, B. (2012). Basic Voice et réduplication en miroir en créole martiniquais. Faits de langue, 38, 193-204.

Jeannot-Fourcaud, B. (2011). Pronoms personnels et réduplication verbale en créole martiniquais : entre appropriation du procès et implication dans le procès, $L a$ linguistique, 47(2), 91-104.

Jeannot-Fourcaud, B. (2003). De l'opposition verbo-nominale en créole martiniquais. Thèse de doctorat non publiée. Paris, Université Paris V.

Klaiman, M. H. (1991a). Grammatical voices. Cambridge : Cambridge University Press.

Klaiman, M. H. (1991b). Control and grammar. Linguistics, 29(4), 623-651.

Klaiman, M. H. (1988). Affectdness and control: a typology of voice systems. Dans M. Shibatani (dir.), Passive and voice (pp. 25-83). Amsterdam/Philadelphia : Benjamins,.

Lazard, G. (2001 [1997]). La typologie actancielle. Dans G. Lazard (dir.), Etudes de linguistique générale: typologie grammaticale (p. 205-216). Rennes: Presses Universitaires de Rennes.

Lazard, G. (1997). Actance, diathèse : question de définition. Pour engager le dialogue avec Igor Mel'cuk. BSL, 92(1), 115-136.

Lyons, J. (1968). Introduction to theorical linguistics. Cambridge : Cambridge University Press.

Mel'cuk, I. (1993). The inflectional category of voice: towards a more rigorous definition. Dans B. Comrie et M. Polinsky (dir.), Causatives and Transitivity (p. 1-46). Amsterdam/Philadelphia : Benjamins.

Parkwall, M. (2003). Reduplication in Atlantic Creoles. Dans S. Kouwenberg (dir.), Twice as meaningful. Reduplication in Pidgins, Creoles and other contact languages (p. 19-36). Westminster Creolistic Series, 8. 
Staudacher-Valliamée, G. (2007). La genèse des créoles de l'Océan Indien : l'éclairage des marqueurs TMA et du dimorphisme verbal en réunionnais. Dans Gadelii K. et ZribiHertz (dir.), Grammaires créoles et grammaire comparative (p. 99-129). Paris : Presses universitaires de Vincennes.

Thibault, A. (2010). «C'est rire qu'il riait » ou l'extraction du prédicat par clivage en français régional antillais. Dans F. Neveu, V. Muni Toke, J. Durand, T. Klingler, L. Mondada

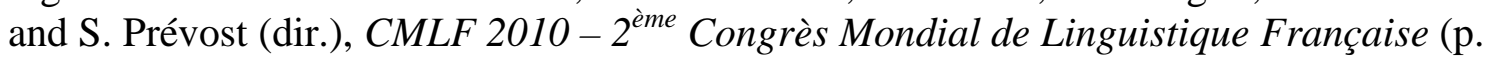
2183-2195). Paris : Institut de Linguistique Française. 


\section{Glossaire}

- ACCOMPLI : l'accompli relève de l'aspect verbal et fait référence au caractère accompli de l'action, c'est-à-dire au fait qu'elle soit achevée. En français le temps correspondant à l'accompli est le passé composé ; on utilise également les termes de parfait ou de perfectif. En créole, la forme qui permet d'indiquer que l'action est achevée est un signifiant zéro ; exemple : $i$ mangé « il a mangé ».

- CO-REFERENCE : le terme de co-référence renvoie au fait que deux unités réfèrent à la même réalité. Ainsi, dans la structure étudiée, le second pronom personnel qui apparaît fait référence à la même réalité que le premier pronom personnel. Par exemple, dans l'énoncé $I$ $k a$ domi domi $a^{\prime} y$, le second pronom personnel $y$ « $3^{\text {ème }}$ personne du singulier » réfère à la même personne que le premier pronom personnel $i$. De même, dans Mari ka alé alé'y, le pronom personnel $y$ est dit en co-référence car il fait référence à la même personne que celle désignée par le prénom Mari et non pas à une autre personne.

- FOCALISATEUR : unité linguistique qui permet la mise en valeur d'un élément de l'énoncé.

- INACCOMPLI : l'unité d'aspect INACCOMPLI se présente formellement, en créole, sous la forme $k a$ et permet d'indiquer le caractère inachevé de l'action ou de l'état.

- IRREALISE : dans cet article, le terme d'irréalisé est utilisé pour rendre compte du fait que le procès est considéré comme irréalisé, n'ayant pas encore eu lieu. En créole, on distingue deux unités de mode irréalisé, ké et kay.

- OCCURRENCE : on parle d'occurrence à chaque fois qu'une même unité linguistique apparaît. Ainsi, lorsqu'il est mentionné «la réduplication du verbal, dont l'une des occurrences est antéposée à l'unité sujet (...)», il s'agit simplement de souligner ici que l'unité verbale apparaît non seulement à sa position habituelle après le sujet, mais également avant le sujet. De même, lorsqu'il est question dans le texte du fait que « la réduplication du verbal (qui) s'accompagne de la postposition d'une occurrence du pronom personnel» (p. 3), il s'agit de signaler qu'après l'unité verbale, apparaît une nouvelle fois le pronom personnel, déjà présent en début d'énoncé.

- PROCES : en linguistique, le terme de procès est utilisé pour référer aussi bien à des actions, processus, états, événements, résultats d'une action, etc. (ex. d'action, ex : marcher, aller; d'état, ex. : être, devenir; de résultat d'une action, ex : savoir, etc). C'est l'acception retenue dans le cadre de cet article. (nota bene: le terme de procès est également utilisé dans certaines traditions grammaticales pour désigner les actions par opposition aux verbes d'état).

- REDUPLICATION : il s'agit de la «répétition» d'une unité ou de plusieurs unités d'une phrase. Selon les langues, les unités rédupliquées peuvent être des syllabes ou bien des unités de rang supérieur, tels que les «mots ». Dans le cas qui nous intéresse, les unités rédupliquées (unités verbales et pronoms personnels) correspondent à des unités, considérées comme des «mots » en grammaire traditionnelle.

- RELATEUR : unité qui permet de lier, de mettre en relation syntaxique deux unités ou deux propositions ; il peut donc s'agir d'une préposition ou d'une conjonction de subordination.

- VERBAL : le terme de verbal est utilisé ici pour désigner les unités qui, en créole, exercent le rôle privilégié de noyau de la phrase, et que l'on pourrait identifier comme étant des unités de même nature que les verbes français. On conserve toutefois le terme de verbal car ces unités présentent des caractéristiques qui les distinguent des verbes tels qu'on les conçoit en linguistique générale, pour des langues comme le français par exemple. 


\section{Annexe}

1) Lis la phrase suivante :

Lésé yo souri souri a-yo, sa pa ka fè ayen

- Cette phrase a-t-elle un sens pour toi ? $\square$ oui

$\square$ non

- Si tu as répondu oui, indique le sens de cette phrase :

- Est-ce que c'est une phrase que tu pourrais toi-même employer dans un contexte donné ? $\square$ oui

- Pourquoi ?

2) Lis la phrase suivante :

Nou té ka domi domi an-nou lè yo sonen

- Cette phrase a-t-elle un sens pour toi ?

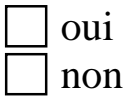

- Si tu as répondu oui, indique le sens de cette phrase :

- Est-ce que c'est une phrase que tu pourrais toi-même employer dans un contexte donné ?

$\square$ oui

- Pourquoi ? 